\title{
Long term evolution of a non-active region sigmoid and its CME activity
}

\author{
A. Glover, L. K. Harra, S. A. Matthews, K. Hori, and J. L. Culhane
}

\author{
Mullard Space Scence Laboratory, University College London, Holmbury St Mary, Dorking, \\ Surrey RH5 6NT, UK \\ e-mail: lkh@mssl.ucl.ac.uk; sam@mssl.ucl.ac.uk; kh@mssl.ucl.ac.uk; jlc@mssl.ucl.ac.uk
}

Received 14 March 2001 / Accepted 3 August 2001

\begin{abstract}
Recent studies have considered sigmoidal features in the Solar corona to possess a high probability of eruption. Unlike these studies which have focussed only on active region sigmoids, the present work centres on a large area which developed $S$-like morphology unconfined by a single active region. The $S$-like feature formed near the central meridian on 8th May 2000 and was observed to erupt, with an associated CME, twice over a period of 3 days. We observe considerable CME and flaring activity in two previous rotations, consistent with the observation of active regions and emerging flux. We illustrate sigmoid formation through the appearance of highly sheared soft X-ray loops overlying an extended filament channel. This arises from the gradual diffusion of flux associated with a large, dispersing active region over the period of three solar rotations. This scenario is indicative of shear build-up within the region over several rotations. The observed CMEs act to remove helicity from the highly sheared sigmoidal feature, finally producing an approximately potential filament channel on 10th May 2000.
\end{abstract}

Key words. sigmoid - Coronal Mass Ejection - flare - helicity

\section{Introduction}

Coronal Mass Ejections (CMEs) are known to originate from both bright, compact active regions and the diffuse quiet corona. As a result, prediction of these events using coronal features remains a topic of intense debate. Recent studies of CME precursors in the solar corona have centred on sigmoidal solar features observed at soft X-ray wavelengths. These studies have suggested that the appearance of a sigmoid within an active region indicates a high probability of eruption (Canfield et al. 1999; Hudson et al. 1998)

Active region sigmoids are observed as transient $S$ (or reverse- $S$ ) shaped features at temperatures of $2 \mathrm{MK}$ and above using the Yohkoh Soft X-ray Telescope (SXT) (Tsuneta et al. 1991). Termed "sigmoidal" by Rust \& Kumar (1996), these features have been interpreted as the projection of helicaly kinked flux ropes onto the solar disk. The observed "twist" of a sigmoidal feature (right handed in the case of $S$-shaped features and left handed where a reverse- $S$ occurs), is indicative of a high degree of magnetic non-potentiality (large free magnetic energy).

Recent studies have proposed the observation of these features on the solar disk to indicate a high probability of eruption to produce flaring and associated CME onset (Canfield et al. 1999; Hudson et al.1998). Further study has illustrated that $S$-shaped features may, on

Send offprint requests to: A. Glover, e-mail: ahg@mssl.ucl.ac.uk occasion, be modeled by potential field models, rather than the expected force-free extrapolations which incorporate the effects of field-aligned currents (Pevtsov et al. 1997). Furthermore, sigmoidal active regions have been shown rarely to comprise of a single feature. Projection effects for collections of smaller soft X-ray loops often result in their appearance as a single $S$ (or reverse$S$ ) shaped structure at Yohkoh/SXT full disk resolution $\left(9.81^{\prime \prime}\right)$ (Glover et al. 2000; Glover et al. 2001). These results highlight the need for a consistent definition of the term "sigmoid", as a quantitative measure of the likelihood of eruption, since it appears that the use of soft X-ray data alone in classification may be misleading.

To date, these studies have concentrated only on sigmoids observed in active regions. Although large scale non-active region sigmoids are known to form (Canfield et al. 2000), to date none have been studied in detail.

This study considers three rotations of a large active region and the surrounding area. This area develops sigmoidal structures during both second and third rotations. The sigmoidal feature formed during the third observed rotation, as illustrated in Fig. 1, provides an example of an extended $S$-shaped structure forming between the dispersed remnants of two earlier active regions. We analyse changes in both coronal and photospheric features in order to gain insight into the processes leading to formation and eruption of the large scale $S$-shaped feature. We also study the CME and flaring activity from the region throughout all three rotations in order to determine 


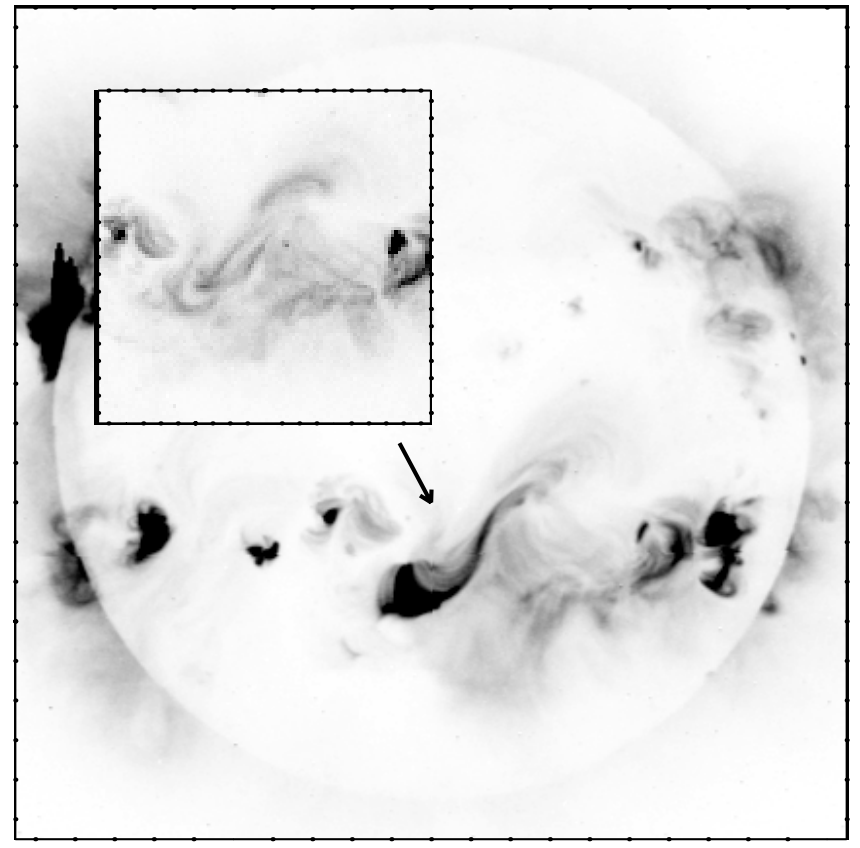

Fig. 1. SXT full disk image illustrating a non-active region sigmoid as it appears to form a single structure at 09:45 UT 8th May 2000 following eruption. Inset illustrates the same region prior to eruption at 04:36 UT.

the variation in activity resulting from shearing motions and emerging twisted magnetic flux. Both processes act to introduce magnetic helicity into the active region field.

\section{Instrumentation}

To date, sigmoidal Solar features have primarily been observed at X-ray temperatures. For the purposes of this investigation, the full range of $2.45^{\prime \prime}, 4.91^{\prime \prime}$ and $9.81^{\prime \prime}$ per pixel spatial resolution observations from the Yohkoh Soft X-ray telescope (SXT) (Tsuneta 1991) have been employed to study the region morphology during each rotation.

In addition to observation of X-ray morphological changes in association with eruptions, photospheric changes were monitored throughout the three observed rotations using full disk magnetograms from the SOHO Michelson Doppler Imager (MDI) (Scherrer et al. 1995). Five-minute averaged magnetograms were available at a cadence of $96 \mathrm{~min}$, with $2^{\prime \prime}$ per pixel spatial resolution and a combined instrumental and background noise level of 8 Gauss (Meunier 1999).

Flare data was obtained from Solar Geophysical Data (SGD: US Dept of Commerce) and refers to 1-8 $\AA$ X-ray observations from the GOES satellite series. GOEs fluxes are classified in terms of $B, C, M$ and $X$ events, representing intensities of $10^{-7} \mathrm{Wm}^{-2}, 10^{-6} \mathrm{Wm}^{-2}, 10^{-5} \mathrm{Wm}^{-2}$ and $10^{-4} \mathrm{Wm}^{-2}$ respectively. Each decade is then split into ten divisions, giving classifications of the form $\mathrm{B} 8$, $\mathrm{M} 4, \mathrm{X} 1$, etc.

In cases where no NOAA active region number had been assigned to a particular GOES event, EIT $195 \AA$ and
SXT full disk data were studied for that period. If flaring was observed nowhere other than the region of interest alone then the event was assumed to originate from that area and thus included in the survey.

CME onset was inferred through observations made by the SOHO Extreme-Ultraviolet Imaging Telescope (EIT) (Delaboudiniere et al. 1995) and the SOHO Large Angle Spectroscopic Coronagraph (LASCO) (Brueckner et al. 1995). Running difference movies were created by subtracting consecutive EIT $195 \AA$ images. These images were available at a cadence of approximately $20 \mathrm{~min}$ with a spatial resolution of approximately $2.62^{\prime \prime}$. In the event that EIT $195 \AA$ data illustrated mass motions associated with the eruption of underlying filament material, EUV dimming and/or a coronal EIT wave from the region under observation (Thompson et al. 1998), a comparison was made with LASCO data.

Preliminary LASCO CME lists (available via ftp from NASA) were checked for coronal mass ejections during each rotation and where the origin of a CME was not listed a height-time fit was applied in order to determine the approximate onset location on the solar disk. If the origin coincided with the region of interest and could be confirmed through SXT observations of flaring and EIT $195 \AA$ dimming and/or coronal EIT wave onset signatures the CME was included in the survey.

The chromospheric data described in Sect. 4 was provided by two sources. H-alpha data for Event A was taken using the HIDA Flare Monitor Telescope (FMT) with a spatial resolution $4.25^{\prime \prime}$. H-alpha data for Event $B$ was provided by the Big Bear Solar Observatory with a spatial resolution $1^{\prime \prime}$.

\section{Long term evolution of AR 8906}

NOAA AR 8906 was initially observed on 9 March 2000. During this rotation it dominated activity on the disk being the largest and most complex active region. Several $\mathrm{M}$ class flares were observed and CME activity was high. Table 1 and Fig. 2 provide comparison between activity associated with the region throughout the three observed rotations. During both March and April rotations a large number of GOES events were observed from the region. This included 6 M-class events during March and 9 Mclass events during April. These rotations also saw the onset of $10 \mathrm{CMEs}$ from the region during the March rotation and 9 CMEs from the region during April. A considerable decrease in activity is observed between April and the region's reappearance in May. Whereas both March and April rotations had produced high levels of CME and flare activity, the May rotation produced only 1 B-class GOES event and 2 CMEs. Following one complete rotation, AR 8906 reappeared on 5 April 2000. The active region itself had been renamed in accordance with the NOAA classification scheme, but will be referred to as AR 8906 throughout this paper for consistency. Figure 3 provides a comparison between full disk SXT and MDI data as the region crosses the central meridian during each 


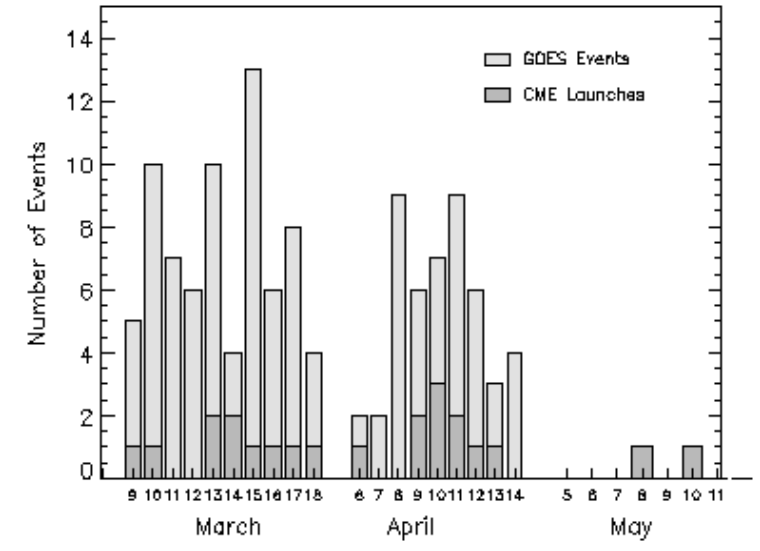

Fig. 2. Distribution of GOES events having magnitude in excess of B1.0 and CME onsets originating from the region illustrated in Figure 3 for the period 9th March to 12th May 2000 .

Table 1. Distribution of GOES events and CME activity from the region of interest over three rotations.

\begin{tabular}{lccccc}
\hline $\begin{array}{l}\text { Date of CM Pass } \\
\text { (2000) }\end{array}$ & \multicolumn{6}{c}{ GOES Events CME Onsets } \\
& X & M & C & B & \\
\hline 13th Mar. & 0 & 6 & 67 & 0 & 10 \\
10th Apr. & 0 & 9 & 39 & 0 & 9 \\
7th May & 0 & 0 & 0 & 1 & 2 \\
\hline
\end{tabular}

rotation. Comparison of Figs. 3b and d illustrates the action of differential rotation on AR 8906. Although the active region itself has decreased in area to approximately $18 \%$ of its spatial extent observed during the March rotation, differential rotation has caused flux to disperse and begin formation of an extended filament channel. SXT data depicting the same time intervals illustrates an increasing tendency of the coronal loops towards an overall $S$-shape in agreement with the hemispheric helicity rule for handedness of the field (Rust \& Kumar 1996). This rule suggests that regions with positive (negative) helicity or $S$-like (reverse- $S$ like) morphology would be more likely to form in the Southern (Northern) hemisphere. Thought to arise from the action of the Coriolis force twisting magnetic flux tubes prior to their emergence at the photosphere, this rule is observed for approximately $65 \%$ of active regions (Canfield et al. 1999; Rust \& Kumar 1996).

The second (April) rotation showed an overall decrease in the level of flaring and CME activity from the area under observation, which now comprised both active region and a filament channel. The most CME-active period during the second rotation coincided with the appearance of a strongly sigmoidal feature illustrated in Fig. 4. Initially observed following a C8.6 class flare from the region on 10th April at 19:11 UT, this sigmoidal feature formed a connection between emerging flux at the centre of AR 8906 and the filament channel extending towards the South West. The observation of a sigmoidal feature during this rotation, indicates a significant increase in helicity content

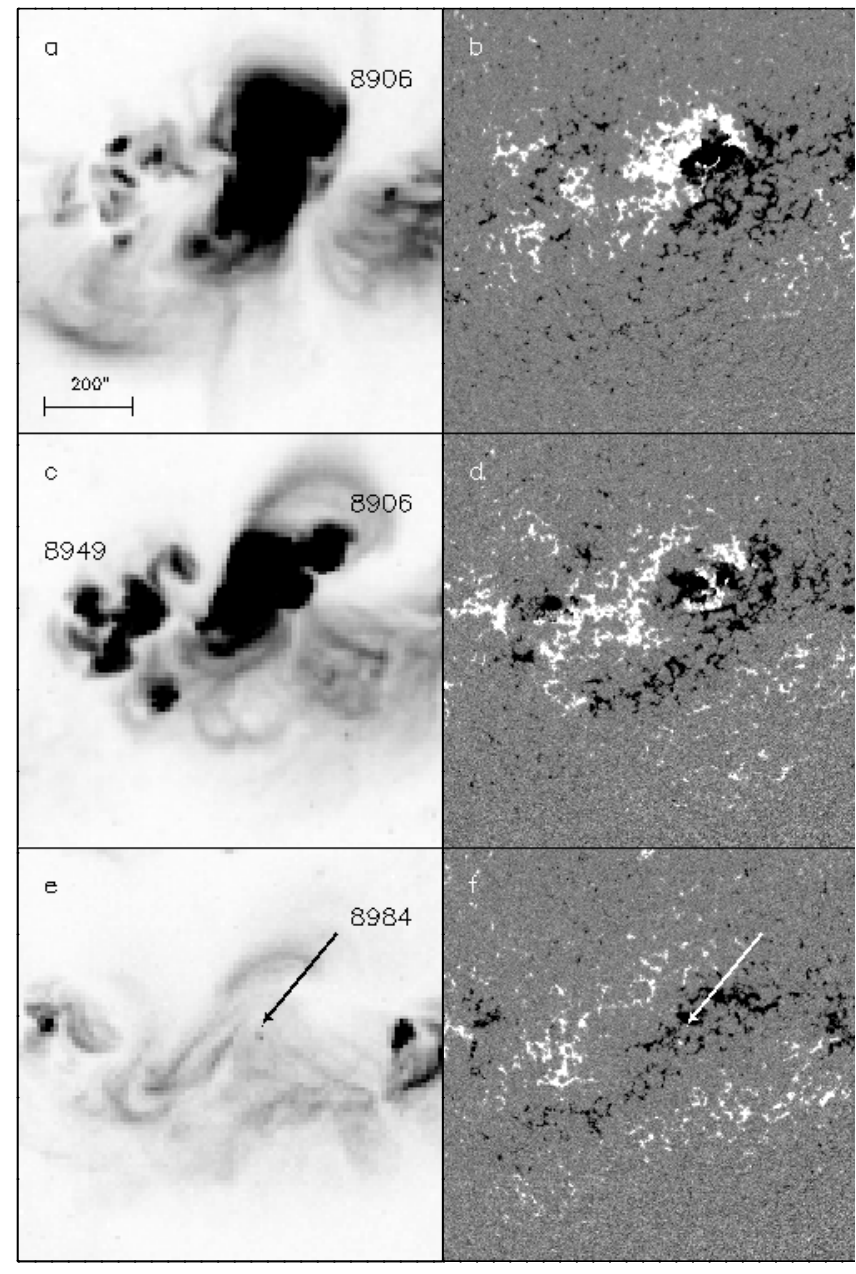

Fig. 3. SXT full disk and MDI full disk observations taken near each central meridian pass of the region; 13th March a), b), 10th April c), d) and 7th May e), f). Images oriented such that Solar North is up and East to the left. Arrows indicate the small emerging flux region classified as AR 8984.

of the diffusing active region 8906 in comparison with that present during the previous (March) rotation, during which no sigmoidal features were observed.

In addition to the action of differential rotation leading to increased levels of shear being applied to active region fields, and thus leading to an overall $S$-shaped character as seen in Fig. 3c, it is also possible that rapidly emerging flux may have contributed to the increased helicity. Emonet \& Moreno-Insertis (1998) illustrated that a flux tube will fragment prior to emergence through the photosphere unless its axis is in possession of some twist. The sigmoidal feature illustrated in Fig. 4 was observed in association with a period of rapidly emerging flux and flaring, thus if the emerging flux were in possession of significant twist on emerging through the photosphere, conservation of helicity states that reconnection with existing active region fields would lead to a proportion of the emerging flux tube twist being transferred to the overlying, longer sigmoidal loop. Thus the distribution of helicity within the active region would be concentrated at this location and the loop's sigmoidal appearance enhanced. 


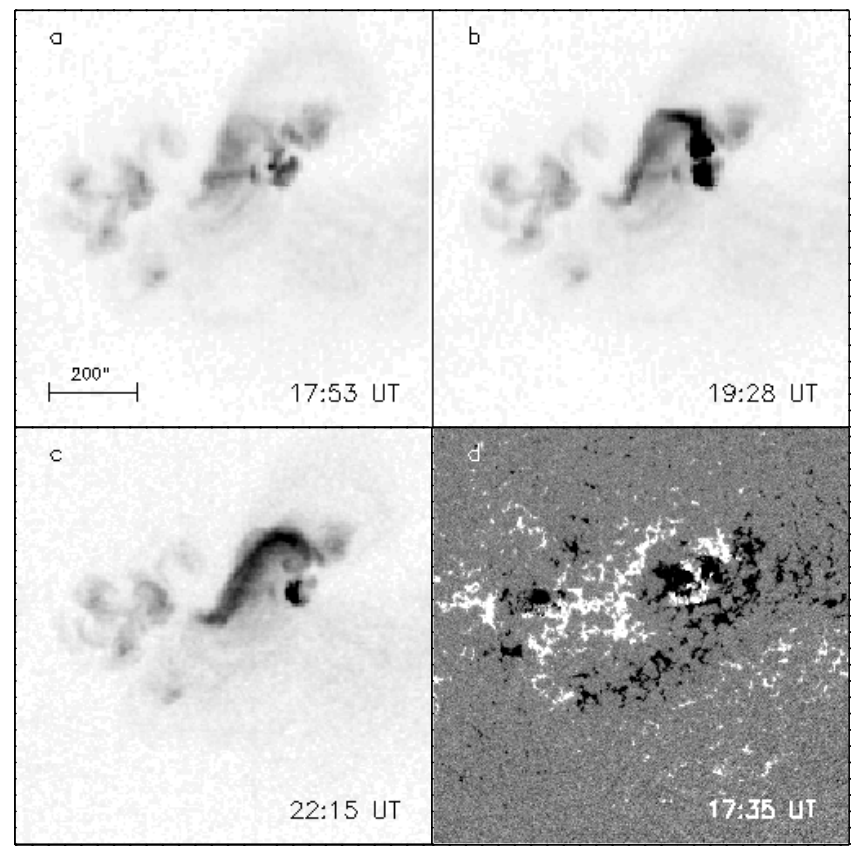

Fig. 4. Full disk $\operatorname{SXT}$ a), b), c) and MDI d) observations taken on 10th April 2000 showing formation of a sigmoidal feature between emerging flux in the NW and the outlying filament channel to the SW. Figure a) illustrates AR 8906 prior to flaring. Figure b) illustrates flux emergence in close association with a C8.6 class flare; peak emission 19:11 UT. This emerging flux region is observed to relax over the following hours to form the sigmoidal feature observed in c). This feature remains visible for approximately 12 hours before fading.

The sigmoid appeared to comprise of a single structure in SXT full disk data, and was observed to gradually fade over a period of approximately 12 hours, rather than disappear in direct association with CME onset. This is suggestive of newly emerged twisted flux reconnecting with field lines already present in the corona, and already in possession of significant helicity. As a result, reconnection would lead to heating of the helical structure to temperatures sufficient to be observed by SXT.

Also coinciding with this active CME period, a further active region, labeled NOAA AR 8949, was observed to emerge in close proximity to the extended filament channel formed by AR 8906. This active region was observed to increase in size throughout the rotation.

The third rotation of AR 8906 and its surrounding strong field regions commenced on 4th May 2000. By this time, the flux associated with both active regions 8906 and 8949 had dispersed almost entirely. MDI observations of the region, shown in Fig. 3f, illustrate an extended filament channel with only a small, positive parasitic polarity region, situated within the filament channel. This parasitic polarity region may be the remnants of AR 8906 . Weak flux emergence within the parasitic region on 5th May 2000 leads to its reclassification as an active region (8984).

Very little flaring activity is observed during this final rotation. A single B6.8 class flare was recorded in
Table 2. Comparison between the two events observed from the sigmoidal region during May 2000.

\begin{tabular}{lll}
\hline & (A) 8th May & (B) 10th May \\
\hline Peak Time (UT) & $06: 23$ & $22: 16$ \\
Flare Class & B6.8 & None \\
CME & Partial Halo & Expanding Loop \\
Filament Eruption & Partial & Yes \\
Flux Cancellation & South of region & Entire Region \\
\hline
\end{tabular}

association with a CME launched from the vicinity of the filament channel. It is at this point that the large scale sigmoidal feature is observed (Fig. 1). One further CME is observed to originate from the highly sheared features overlying the neutral line during the remaining days of this rotation. Taking place on 10th May 2000, this final CME results in the appearance of a soft X-ray arcade exhibiting a large reduction in shear when compared with the previously observed highly sheared structure. Both these events are described in detail in the following section.

\section{Activity during rotation 3, May 2000: Formation and evolution of non-AR sigmoidal feature}

During the third rotation of AR 8906, two eruptions took place, as described in Table 2. The first event, a GOES Xray class B6.8 two-ribbon flare, peaked at 06:23 8th May 2000. This occurred as the now diffuse filament channel passed close to central meridian. In this case, an increase in soft X-ray intensity was observed to originate from only the southern half of the region.

Figure 5a illustrates that, prior to eruption, soft Xray features overlying the extended filament channel comprise many diffuse loops arranged in an overall $S$-shape. Following eruption however, SXT full disk data $\left(9.81^{\prime \prime}\right)$, illustrated in Fig. 5b, suggests formation of a single twisted flux rope in the following hours. Comparison with EIT $195 \AA$ data, pictured in Figs. 5c and d, illustrates the bright, curved Southern tail of the sigmoidal feature to be consistent with the appearance of a post-flare arcade. These loops are observed to cool and fade over the hours following eruption. Consequently, the apparently sigmoidal feature also fades.

A faint partial halo CME was observed by the LASCO/C2 coronagraph. A height time extrapolation confirms onset time to be consistent with this event.

H-alpha observations made by the HIDA Flare Monitor Telescope (FMT) during the eruption, suggest the occurrence of a two-part filament eruption beneath the sigmoidal feature. Figures 5e and f illustrate disappearance of the curved Southern half of the filament during the event, with the formation of bright footpoints consistent with observation of a bright arcade in EIT $195 \AA$ and SXT data. Extensive mass movement is observed in the upper half of the filament, although no complete disappearance and/or eruption takes place. 


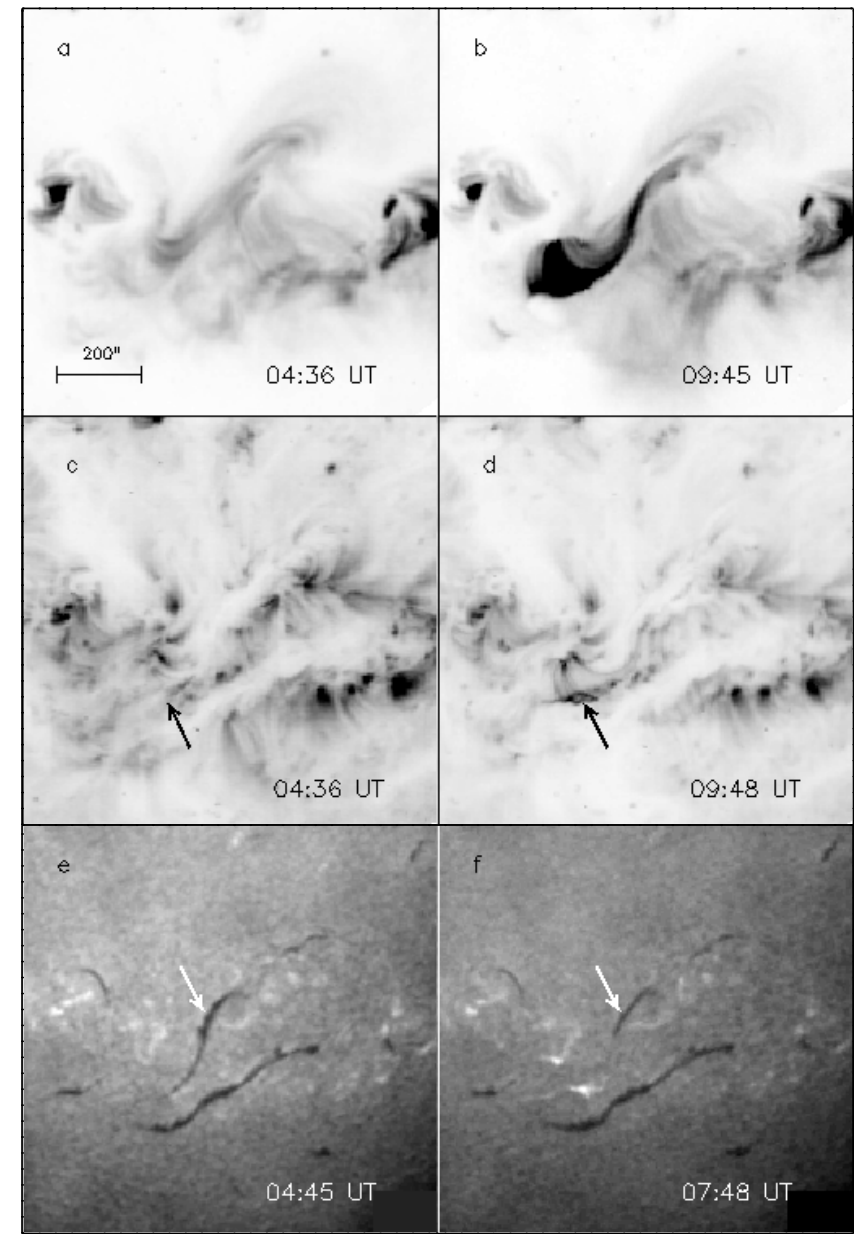

Fig. 5. Comparison of SXT full disk data prior to a) and following b) eruption on 8th May 2000 with EIT $195 \AA$ c) and d) and H-alpha e) and f) data of the same period. Arrows in Figs. c) and d) illustrate observation of post-flare loops in the Southern half of the filament channel following eruption. Arrows in Figs. e) and f) indicate the $S$-shaped filament underlying the soft X-ray arcade. The second filament to the South remains unchanged throughout the eruption.

Following this event, the sigmoidal feature fades gradually in SXT data until 10th May 2000. Figure 6 illustrates the soft X-ray morphology of the region on 10th May 2000. No increases in soft X-ray intensity have been observed since formation of the sigmoidal feature on 8th May 2000. At this stage, the region under observation has rotated towards the limb and sigmoidal morphology is no longer clearly distinguishable. Figure 7a illustrates the region's appearance in soft X-ray data as a collection of highly sheared loops overlying the neutral line prior to eruption.

Commencing at 10th May 2000 16:15:27 UT, and illustrated by Figs. 7b-d, soft X-ray emission from the sheared arcade begins a gradual increase. No GOES class flare is recorded in association with this event. However, the arcade is observed to expand and brighten, with a decrease in shear angle of approximately 25 degrees observed. This is suggestive of helicity removal from the sheared arcade in association with CME onset. Maximum emission is

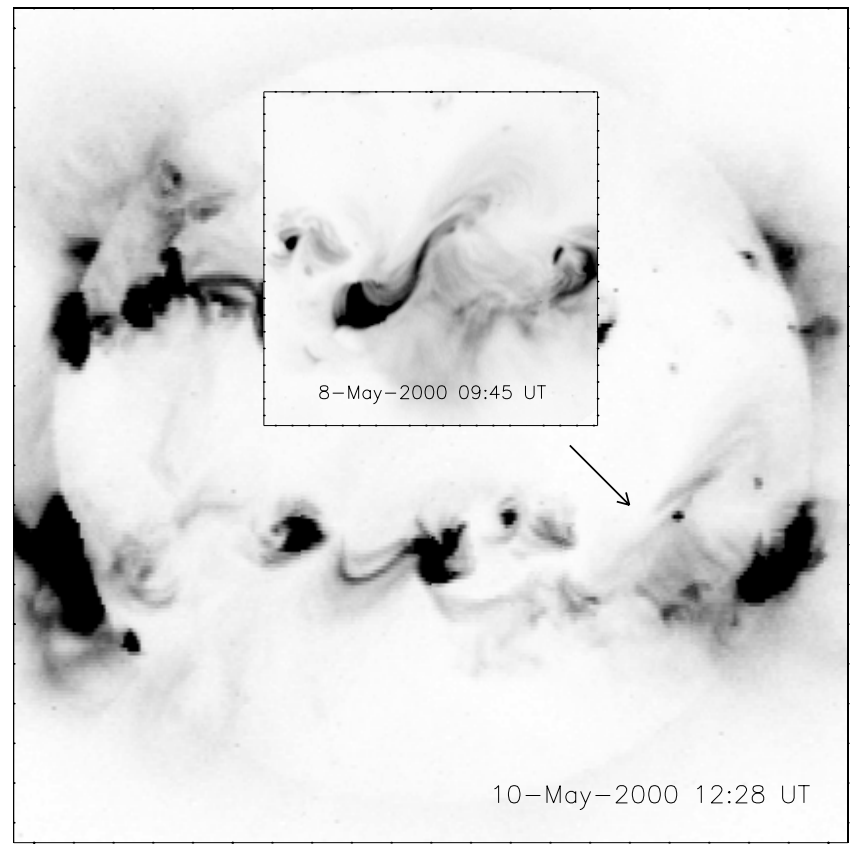

Fig. 6. SXT full disk data providing a comparison soft X-ray morphology of the region under investigation following sigmoid formation on 8th May 2000 (inset) and after a further two days rotation on the 10th May 2000 (main image).

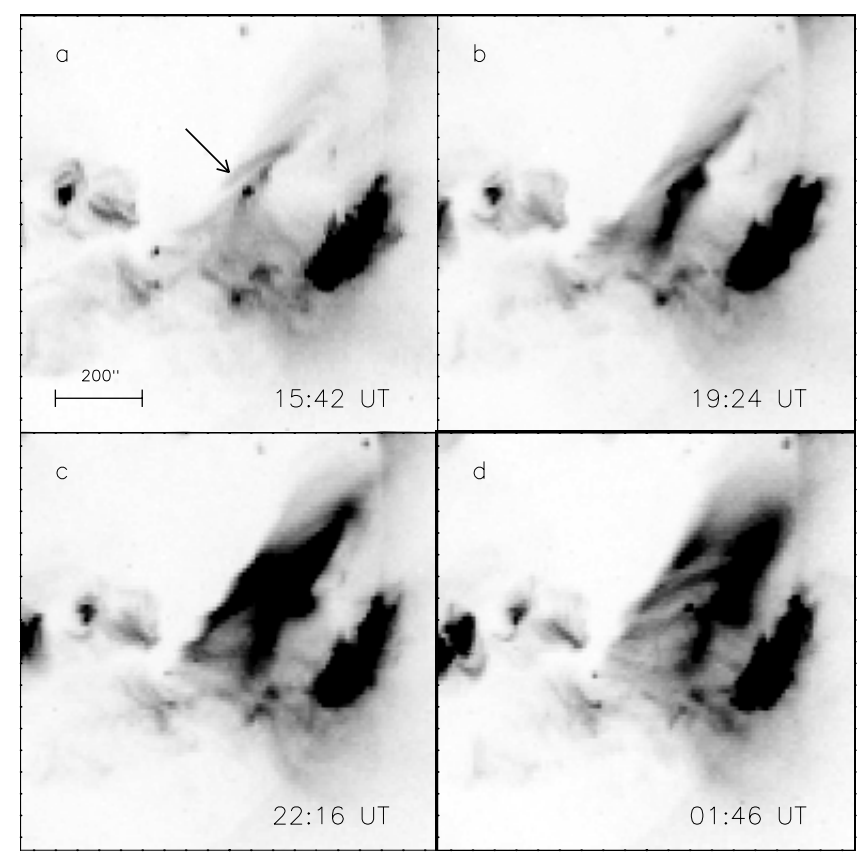

Fig. 7. SXT full disk data showing evolution of the region as it erupts on 10th May 2000. Prior to the eruption a) the region is comprised of a highly sheared arcade, the location of which is indicated by the arrow, b) and c) show a gradual rise and expansion of this arcade until, following a filament eruption, a reduction in shear angle of 25 degrees is observed $\mathbf{d}$ ).

observed from the arcade at 22:16:31 UT directly following Yohkoh night.

A loop-like CME was observed in the South West quadrant of the LASCO/C2 coronagraph at 19:26 UT. 


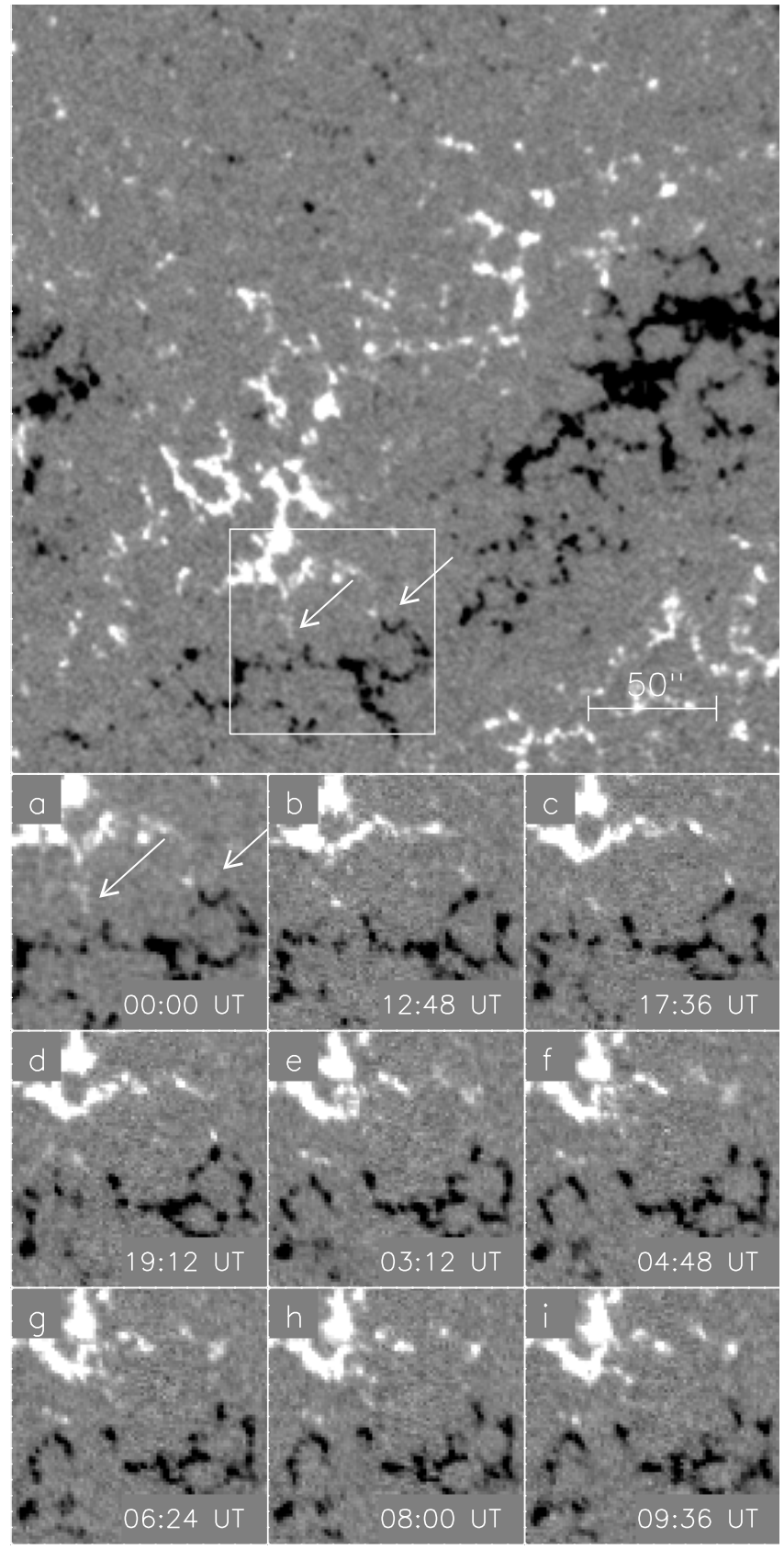

Fig. 8. SOHO/MDI observations illustrating flux cancellation near the Southern edge of the filament channel in association with flaring and CME onset on 8th May 2000. Image sequence begins on 7th May and continues until after CME onset on 8th May. Arrows indicate clearest examples of flux cancellation.

A height time extrapolation confirms onset time to be consistent with the gradually rising soft X-ray arcade.

SoHO/MDI observations of the filament channel prior to and during each eruption are shown in Figs. 8 and 9. These figures illustrate sample positive and negative magnetic fragments drifting towards the centre of the channel and canceling. Cancellation was observed in the Southern half of the region, consistent with the observed flare locality, during the first event (Fig. 8) and along the full length of the filament channel prior to and during the

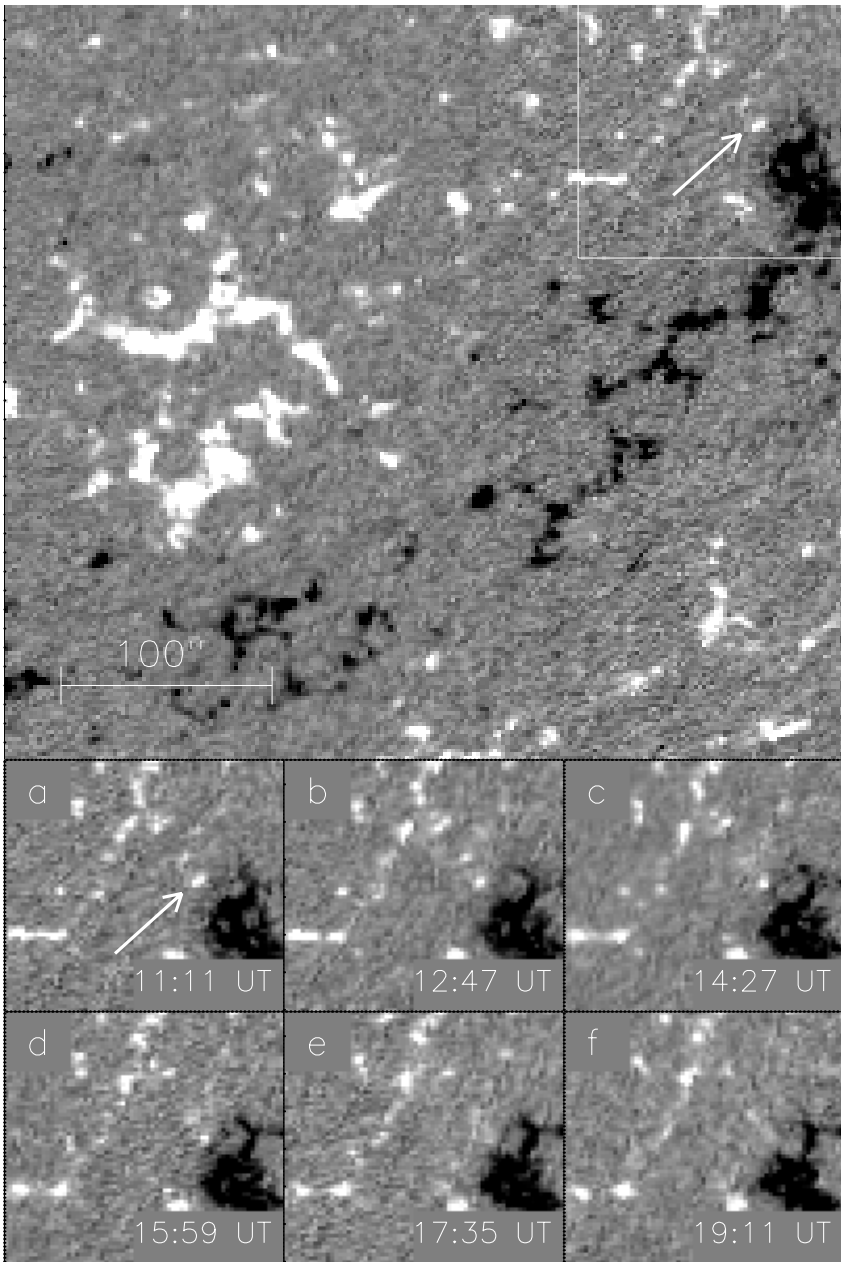

Fig. 9. SOHO/MDI observations illustrating flux cancellation along the neutral line prior to and during the filament eruption observed on 10th May 2000. In this case, cancellation takes place along the entire length of the filament channel. However, only the clearest example is illustrated here. Arrow indicates canceling polarity regions.

second event (Fig. 9). This is suggestive of reconnection beneath the suspended filament plasma creating helical magnetic fields from photospheric shearing motions acting on the already sheared overlying arcade (van Ballegooijen \& Martens 1989). The footpoints of smaller loops, formed through reconnection, are observed to cancel and disappear as the loop sinks beneath the photosphere owing to magnetic tension and forces associated with strong downward curvature of small loops. While this is taking place, the larger helical field erupts with the prominence material. If this is the case, the CME may provide a mechanism through which helicity, arising through the influence of photospheric shear on the filament channel, can be removed from the region. van Ballegooijen \& Martens also found that, as the number of helical field lines formed through this process increased, the height at which the field remained steady also increased. Therefore, it may be the case that this build up of helicity localised in the vicinity of the prominence itself, might have a destabilising effect on the prominence material, leading to eruption. 


\section{Discussion}

This study follows the evolution of an active region through three rotations between March and May 2000. Initially this active region was highly complex and dominated activity on the disk throughout the March rotation. The main active region reduced in field strength prior to its reappearance in April as the action of differential rotation caused the active region flux to disperse and spread out with the formation of an extended filament channel. During the second rotation an $S$-shaped structure connecting emerging flux within AR 8906 to the outlying filament channel was observed for a period of approximately 12 hours. This coincided with an increase in CME activity and the emergence of a second active region to the South East of the dispersing NOAA AR 8906. Magnetic flux associated with both active regions was observed to have undergone further dispersion prior to the third rotation, leaving primarily only single polarities to either side of an extended neutral line.

Figure 3 illustrates an increase in shear over the course of 3 rotations. Soft X-ray data illustrates that the dispersing AR 8906 tends towards an overall $S$-shape during the second and third rotations. During the final two rotations, individual sigmoidal "features" were observed. The first of these extended approximately $150^{\prime \prime}$ in $[x, y]$ coordinates and formed a connection between emerging flux within active region 8906 and part of the outlying filament channel. This feature was relatively short lived; disappearing after approximately 12 hours. The second sigmoidal feature was observed during the third rotation, as a highly sheared arcade joining the decayed remnants of two active regions on either side of the main neutral line and spanning approximately $450^{\prime \prime}$ in $[x, y]$ coordinates. This feature formed to the South of the equator above an extended neutral line, forming a connection between a small active region (8984) and a region of positive flux to the South East. The sigmoid erupted soon after formation.

Observations of AR 8906 and its surrounding area show flux emergence taking place during all three rotations. Although weak during the third rotation, March and April observations illustrate strong flux emergence in agreement with the observed high levels of flaring. Furthermore, the $S$-shaped structure observed on 11th April forms during a period of strong flux emergence. In this case, reconnection between emerging flux and existing active region fields acts to highlight a single twisted $S$-shaped structure. This provides evidence of an increased helicity content present within the region fields during the second rotation as compared to first (March) rotation during which no helical or $S$-shaped structures were observed.

Recent studies (e.g. Emonet \& Moreno-Insertis 1998] have indicated that emerging flux must be in possession of some twist in order to prevent fragmentation below the photosphere. Thus, emerging flux must possess some twist, which through helicity conservation would be transferred on reconnection to the longer overlying field. Thus, helicity could be added to the region through both differential rotation and emerging flux during all three rotations, but removed through CME onset. This would be consistent with continued CME activity during the third (May) rotation, even though there is a significant reduction in flux emergence following the second rotation.

Previous studies have proposed both differential rotation (DeVore 2000) and the emergence of flux tubes already in possession of significant twist (Pevtsov et al. 1995) to make strong contributions to the total helicity of coronal fields within active regions. Observation of AR 8906 over three rotations confirms this to be the case as emerging flux is observed during each rotation, and the effects of differential rotation on the region are clear as the active region flux is observed to disperse and gradually merge with surrounding weaker field regions.

In order to estimate the increase in footpoint separation likely to arise in the area under consideration over three rotations through the action of differential rotation alone, the difference between rotation rates experienced at the Northern and Southern extents of the area $\left(\Delta \omega_{\mathrm{NS}}\right)$ is calculated. This rate is then evaluated over the number of days separating each central meridian pass $(\tau)$, giving an estimated increase in footpoint separation due to differential rotation. The increase in longitudinal extent predicted by calculation $(\Delta \times($ calc $))$ is then compared to the observed increase in longitudinal spread of the region $(\Delta \times($ obs $))$.

A simple expression for the latitude dependent rate of solar rotation is as follows:

$\omega(\psi)=\omega_{\mathrm{o}}-\omega_{2} \sin ^{2}(\psi)\left[{ }^{\mathrm{o}} \mathrm{day}^{-1}\right]$

where $\omega_{0}$ represents the maximum rotation rate at the solar equator, $\psi$ the latitude at which the rotation rate is to be evaluated and $\omega_{2}$ is taken to be 2.77 day $^{-1}$ following Newton \& Nunn (1951). This expression can be modified to calculate the difference in rotation rate between two latitudes $\Delta \omega_{\mathrm{NS}}$ as follows:

$$
\Delta \omega_{\mathrm{NS}}=\omega\left(\psi_{\mathrm{N}}\right)-\omega\left(\psi_{\mathrm{S}}\right)=\omega_{2}\left(\sin ^{2}\left(\psi_{\mathrm{N}}\right)+\sin ^{2}\left(\psi_{\mathrm{S}}\right)\right)
$$

where $\psi_{\mathrm{N}}$ and $\psi_{\mathrm{S}}$ denote Northern and Southern extents of the region prior to rotation. An estimate of the increased separation resulting from the action of differential rotation alone can then be expressed as:

$\Delta x($ calc $\left.)=\left(\Delta \omega_{\mathrm{NS}}\right) \tau \quad{ }^{\circ}\right]$

where $\tau$ denotes number of days between each central meridian crossing.

Table 3 illustrates the diffusion suffered by AR 8906 over the course of three rotations. Comparison with the increased separation predicted by the action of differential rotation alone shows that although this mechanism undoubtedly contributes significantly to the shear increase within the region, other factors including the observed emerging flux, flux cancellation and flux diffusion owing to the action of supergranular flow at the photosphere (van Ballegooijen 1999) must also contribute to the increase. 
Table 3. Comparison between observed increase in longitudinal extent of AR 8906 over three rotations $(\Delta \times($ obs $))$ and values estimated based on the action of differential solar rotation alone $(\Delta \times($ calc $))$.

\begin{tabular}{lcccc}
\hline $\begin{array}{l}\text { CM Pass } \\
(2000)\end{array}$ & $\begin{array}{c}\text { Co-ords } \\
\text { (North) }\end{array}$ & $\begin{array}{c}\text { Co-ords } \\
(\text { South })\end{array}$ & $\begin{array}{c}\Delta x(\text { obs }) \\
\left({ }^{\circ}\right)\end{array}$ & $\begin{array}{c}\Delta x(\text { calc }) \\
\left({ }^{\circ}\right)\end{array}$ \\
\hline 13th Mar. & S09W09 & S27E07 & 16 & - \\
10th Apr. & S08W12 & S33E19 & 31 & 9 \\
7th May. & S09W16 & S32E24 & 40 & 17 \\
\hline
\end{tabular}

Comparison of CME and flaring activity within the region shows a decrease in flaring over all three rotations observed here. However, the number of CMEs associated with the region remains high during the second rotation. The third rotation is the least active of the three, with only one B class flare being produced. It is interesting to note that this event originates from the Southern half of the sigmoidal region, rather than the previously active Northern half which had contained the remnants of AR 8906. During this final rotation, the number of CMEs exceeds the number of flares as the region continues to disperse.

The principle of helicity conservation states that, in the corona, helicity may be approximately conserved when magnetic field topology is not i.e. during periods of reconnection or confined flaring (Berger 1999). If this were the case, flaring alone would be unable to remove twist from the solar field. It has been proposed through both application of theoretical models and observation that the CME may act as a valve through which helicity is removed from the corona (van Driel-Gesztelyi et al. 1999; Low 1996).

We infer helicity removal in the present study by comparison of the CME events of 8th May 2000 and 10th May 2000. Observation of the $S$-shaped feature on 8th May 2000 as a result of CME onset may be interpreted as an indication that only a fraction of the total helicity has been removed from the filament channel by the CME. The sheared post-flare arcade giving the appearance of an $S$-shaped structure fades, until erupting two days later to produce an arcade with significantly reduced shear angle, thus indicating that a significant amount of helicity has finally been removed from the region and the resulting magnetic non-potentiality has been reduced.

\section{Conclusions}

This study provides the first detailed study into the formation and evolution of a large scale, non-active region sigmoidal feature observed in soft X-ray data.

The overall $S$-shaped nature of AR 8906 is observed to develop over the course of three rotations. This increase in sheared appearance is believed to form as a result of an increase in helicity contained within the region and provided by the combination of several factors including differential rotation and emerging flux already in posses- sion of significant shear prior to its emergence through the photosphere.

The $S$-like appearance is then reduced through the continued high CME activity of the region. Sigmoidal morphology is finally removed through continued CME activity during the third, final rotation when flaring activity has almost ceased.

Acknowledgements. A. G. would like to thank all those who assisted with the organisation and running of JOP 120 through which the "sigmoid" observations detailed here were carried out, L. van Driel Gesztelyi for helpful discussions, Satoru UeNo for providing HIDA/FMT data and BBSO for providing H-alpha data. A. G. would also like to thank PPARC for a Ph.D. Studentship and L. K. H. also acknowledges PPARC for an advanced fellowship. Yohkoh/SXT data was provided by the Solar UK Research Facility (SURF).

\section{References}

Berger, M. A. 1999, in Magnetic Helicity in Space and Laboratory Plasmas, ed. M. R. Brown, R. C. Canfield \& A. A. Pevtsov, Geophys. Monogr. Ser., 111, 1

Brueckner, G. E., et al. 1995, Solar Phys, 162, 357

Canfield, R. C., Hudson, H. S., \& Pevtsov, A. A. 2000, in Special Issue of IEEE Trans. on Plasma Science, 2000

Canfield, R. C., Hudson, H. S., \& McKenzie, D. E. 1999, Geophys. Res. Lett., 26, 627

Delaboudiniere, J.-P., Artzner, G. E., Brunaud, J., et al. 1995, Solar Phys., 162, 291

DeVore, C. R. 2000, ApJ, 539

Emonet, T., \& Moreno-Insertis, F. 1998, ApJ, 492, 804

Glover, A., Ranns, N. D. R., Harra, L. K., \& Culhane, J. L. 2000, Geophys. Res. Lett., 27(14), 2161

Glover, A., Ranns, N. D. R., Brown, D. S., et al. 2001, JASTP, submitted.

Hudson, H. S., Lemen, J. R. St., Cyr, O. C., Sterling, A. C., \& Webb, D. F. 1998, Geophys. Res. Lett., 14, 2481

Low, B. C. 1996, Solar Phys., 167, 217

Meunier, N. 1999, ApJ, 515, 801

Newton, H. W., \& Nunn, M. L. 1951, MNRAS, 111, 413

Pevtsov, A. A., Canfield, R. C., \& McClymont, A. N. 1997, ApJ, 481, 973

Pevtsov, A. A., Canfield, R. C., \& Metcalf, T. R. 1995, ApJL, 440, 109

Rust, D. M., \& Kumar, A. 1996, ApJL, 464, 199

Scherrer, P. H., Bogart, R. S., Burl. R. I., et al. 1995, Solar Phys., 162, 129

Thompson, B. J., Plunkett, S. P., Gurman, J. B., et al. 1998, Geophys. Res. Lett., 25, 2461

Tsuneta, S., Acton, L., Brurer, M., et al. 1991, Solar Phys., 136,37

van Ballegooijen, A. A. 1999, in Magnetic Helicity in Space and Laboratory Plasmas, ed. M. R. Brown, R. C. Canfield \& A. A. Pevtsov, Geophys. Monogr. Ser., 111, 213

van Ballegooijen, A. A., \& Martens, P. C. H. 1989, ApJ, 343, 971

van Driel-Gesztelyi, L., Mandrini, C. H., Thompson, B., et al. 1999, in 3rd Advances in Solar Phys., ed. B. Schmieder, A. Hofmann, \& J. Staude, ASP Conf. Ser. 184, 302 